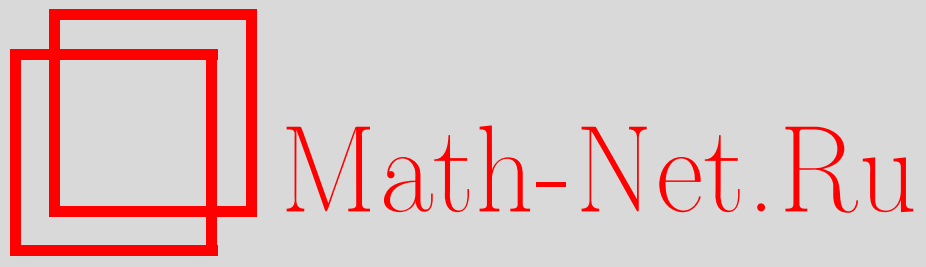

A. М. Гомилко, Об обратном операторе генератора ограниченной $C_{0}$-полугруппы, Функи. анализ и его прил., 2004, том 38, выпуск 4, 6-12

DOI: https://doi.org/10.4213/faa122

Использование Общероссийского математического портала MathNet.Ru подразумевает, что вы прочитали и согласны с пользовательским соглашением

http://www . mathnet.ru/rus/agreement

Параметры загрузки :

IP : 54.198 .64 .247

26 апреля 2023 г., 17:40:53

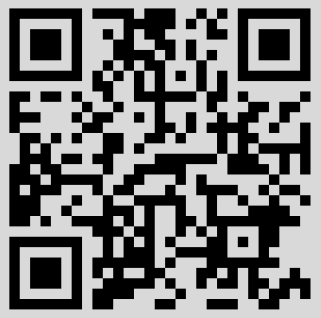


Функииональньй анализ и его приложения

2004, т. 38, вып. 4, с. 6-12

УДК 517.9

\title{
Об обратном операторе генератора ограниченной $C_{0}$-полугруппы
}

\author{
(c) 2004. А. М. Гомилко
}

\author{
Посвящается В. Б. Лидскому \\ в связи с его восъмидесятилетием
}

1. Всюду далее $B$ - банахово пространство с нормой $\|\cdot\|$ и $E=E(B)-$ множество линейных плотно определенных замкнутых операторов, действующих в $B$. Через $D(A)$ мы обозначаем область определения оператора $A \in E$, а через $\sigma(A)$ - его спектр, $I$ - единичный оператор и $R(A, \lambda)=(A-\lambda I)^{-1}, \lambda \notin \sigma(A)$, - резольвента оператора $A$. Далее, $E_{-}=E_{-}(B)-$ множество операторов из $E$ со спектром, расположенным в полуплоскости $\operatorname{Re} \lambda \leqslant 0$. Если $x \in B$ и $y \in B^{*}$, где $B^{*}$ - сопряженное к $B$ банахово пространство с нормой $\|\cdot\|_{*}$, то через $(x, y)$ обозначаем действие функционала $y$ на $x$.

Напомним необходимые для дальнейшего сведения из теории полугрупп операторов ([1, гл. 9] и [2, гл. 8]). Семейство $T(t), t \geqslant 0$, линейных ограниченных операторов, действующих в $B$, образует $C_{0}$-полугруппу операторов, если $T(0)=I, T\left(t_{1}+t_{2}\right)=T\left(t_{1}\right) T\left(t_{2}\right)\left(t_{1}, t_{2} \geqslant 0\right)$ и $\|T(t) x-x\| \rightarrow 0$ при $t \rightarrow 0$ для любого $x \in B$. Всюду далее под полугруппой будем понимать $C_{0}$-полугруппу операторов. Генератор (производящий оператор) $A \in E$ полугруппы $T(t)$ определяется формулой

$$
A x=\lim _{t \rightarrow 0} \frac{T(t) x-x}{t}, \quad x \in D(A) .
$$

При этом для соответствующей полугруппы будем пользоваться обозначением $T(t)=e^{t A}, t \geqslant 0$. Полугруппа $T(t)$ называется равномерно ограниченной, если найдется такая постоянная $M \geqslant 1$, что $\|T(t)\| \leqslant M, t \geqslant 0$. Через $\mathscr{G}=\mathscr{G}(B)$ обозначим множество генераторов равномерно ограниченных полугрупп линейных операторов, действующих в $B$.

Пусть $\mathscr{H}(\theta)[1$, гл. 9, §1] - множество генераторов, порождающих голоморфные (аналитические) полугруппы в секторе $\Sigma_{\theta}=\left\{z=r e^{i \phi}: 0<r<\infty,|\phi|<\theta\right\}$, $\theta \in(0, \pi / 2]$, являющиеся равномерно ограниченными в произвольном секторе $\Sigma_{\theta_{0}}, \theta_{0} \in(0, \theta)$. Через $\mathscr{H}_{0}$ обозначим множество всех операторов вида $A=$ $A_{0}+\beta I$ с некоторыми $A_{0} \in \mathscr{H}(\theta)$ и $\beta \geqslant 0, \theta \in(0, \pi / 2]$. Множество $\mathscr{H}_{0}$ состоит из генераторов голоморфных в некотором секторе $\Sigma_{\theta}$ (в случае $\beta>0$ квазиограниченных по терминологии $[1$, гл. $9, \S 1.4])$ полугрупп.

Известно [3,4], что если $A \in \mathscr{H}(\theta)$ и существует обратный оператор $A^{-1} \in E$ (т. е. $\operatorname{ker} A=\{0\}$ и область значений оператора $A$ является плотной в $B$ ), то $A^{-1}$ также принадлежит $\mathscr{H}(\theta)$ и, в частности, $A^{-1} \in \mathscr{G}$. Действительно $[1,2]$, включение $A \in \mathscr{H}(\theta)$ эквивалентно тому, что резольвента $R(A, \lambda)$ существует 
при $\lambda \in \Sigma_{\pi / 2+\theta}$ и допускает там оценку

$$
\|R(A, \lambda)\| \leqslant \frac{c_{\phi}}{|\lambda|}, \quad \lambda \in \Sigma_{\pi / 2+\phi}, \quad \text { для любого } \phi \in(0, \theta) .
$$

Поэтому, если $A \in \mathscr{H}(\theta)$ и $A^{-1} \in E$, то при $\lambda \in \Sigma_{\pi / 2+\theta}$ имеем

$$
\left(A^{-1}-\lambda I\right)^{-1}=-\lambda^{-1}\left[I+\lambda^{-1} R\left(A, \lambda^{-1}\right)\right]
$$

и тогда из (1), (2) немедленно вытекает оценка $\left\|R\left(A^{-1}, \lambda\right)\right\| \leqslant\left(1+c_{\phi}\right)|\lambda|^{-1}$, $\lambda \in \Sigma_{\pi / 2+\phi}$, для любого $\phi \in(0, \theta)$, т. е. $A^{-1} \in \mathscr{H}(\theta)$.

В данной статье утверждение

$$
A \in \mathscr{H}(\theta), A^{-1} \in E \Longrightarrow A^{-1} \in \mathscr{G}
$$

распространяется на более широкий класс генераторов равномерно ограниченных полугрупп. Доказано, что если $A \in \mathscr{G}$ и для любых $x \in B, y \in B^{*}$ найдутся такие числа $\sigma_{0}>0, r_{2}>0, r_{1} \geqslant r_{2}$ и конечная положительная мера $\mu(t)$ на $\mathbb{R}$ (зависящие от пары $x, y$ ), что справедливы оценки

$$
\begin{gathered}
\sigma \int_{\sigma-i \infty}^{\sigma+i \infty}\left|\left(R^{2}(A, \lambda) x, y\right)\right||d \lambda|<\infty, \quad \sigma \in\left(0, \sigma_{0}\right), \\
\left|\left(R^{2}(A, \lambda) x, y\right)\right| \leqslant \int_{-\infty}^{\infty} \frac{d \mu(t)}{|i t-\lambda|^{2}}, \quad \operatorname{Re} \lambda>0,|\lambda| \in\left(0, r_{2}\right) \cup\left(r_{1}, \infty\right),
\end{gathered}
$$

и существует оператор $A^{-1} \in E$, то $A^{-1} \in \mathscr{G}$ (если $r_{1}=r_{2}$ либо $B=H$ - сепарабельное гильбертово пространство, то условие (3) можно опустить). В качестве следствия получено, что если $B=H$ - сепарабельное гильбертово пространство и $A \in \mathscr{H}_{0}, A^{-1} \in \mathscr{H}_{0}$, то полугруппы $e^{t A}$ и $e^{t A^{-1}}$ могут быть равномерно ограниченными при $t \geqslant 0$ лишь одновременно.

Далее используется следующее достаточное условие принадлежности оператора $A$ из $E_{-}$множеству $\mathscr{G}[5]:$ если для любых $x \in B, y \in B^{*}$ выполняется оценка

$$
\sup _{\sigma>0} \sigma \int_{\sigma-i \infty}^{\sigma+i \infty}\left|\left(R^{2}(A, \lambda) x, y\right)\right||d \lambda|<\infty
$$

то $A \in \mathscr{G}$. В случае сепарабельного гильбертова пространства это условие является и необходимым для того, чтобы $A \in \mathscr{G}$. Это вытекает из неравенства

$$
\sigma \int_{\sigma-i \infty}^{\sigma+i \infty}\|R(A, \lambda) x\|^{2}|d \lambda|=2 \pi \sigma \int_{0}^{\infty} e^{-2 \sigma t}\left\|e^{t A} x\right\|^{2} d t \leqslant \pi M^{2}\|x\|^{2}, \quad \sigma>0,
$$

справедливого для оператора $A \in \mathscr{G}(H)$, аналогичного неравенства для резольвенты оператора $A^{*} \in \mathscr{G}(H)$ и неравенства Коши-Буняковского.

2. Отметим, что выполнение условий (3) и (4) при $|\lambda|>r_{1}$ влечет за собой выполнение условия (5) и, значит, $A \in \mathscr{G}$. Это вытекает из ограниченности резольвенты $R(A, \lambda)$ на компакте $\left\{\lambda: \operatorname{Re} \lambda \geqslant \sigma_{0},|\lambda| \leqslant r_{1}\right\}$ (в случае $\left.\sigma_{0}<r_{1}\right)$ и простой оценки

$$
\int_{\sigma-i \infty}^{\sigma+i \infty}\left\{\int_{-\infty}^{\infty} \frac{d \mu(t)}{|i t-\lambda|^{2}}\right\}|d \lambda| \leqslant \int_{-\infty}^{\infty}\left\{\int_{-\infty}^{\infty} \frac{d s}{(s-t)^{2}+\sigma^{2}}\right\} d \mu(t)=\frac{\pi}{\sigma} m<\infty
$$


где

$$
m=\int_{-\infty}^{\infty} d \mu(t)
$$

ЗАмечание. Так как резольвента $R(A, \lambda)$ оператора $A \in E_{-}$ограничена по норме на любом компакте $K$, лежащем в открытой полуплоскости $\operatorname{Re} \lambda>0$, то, не уменьшая общности, можно считать, что интегральная оценка (4) справедлива в области

$$
\Omega\left(b ; r_{0}\right)=\left\{\lambda \in \mathbb{C}: \operatorname{Re} \lambda>0,|\lambda \pm i b|>r_{0}\right\}
$$

для некоторых $b>0$ и $r_{0} \in(0, b)$ с возможной заменой меры $\mu(t)$ в $(4)$ на меру $\mu(t)+c\|x\|\|y\|_{*} \delta_{0}$, где $c>0$ - некоторая постоянная, а $\delta_{0}$ - единичная дискретная мера, сосредоточенная в точке $t=0$.

ЛЕмма 1. Пусть резольвента оператора $A \in E_{-}$удовлетворяет условию (4) при $\lambda \in \Omega\left(b ; r_{0}\right)$. Тогда для любых $x \in B, y \in B^{*}$ справедливы оценки

$$
|(R(A, \lambda) x, y)| \leqslant \frac{\pi}{2} \int_{-\infty}^{\infty} \frac{d \mu(t)}{|i t-\lambda|}, \quad \lambda \in \Omega\left(b ; r_{0}\right) .
$$

Доказательство. Из (4) следует, что $\left|\left(R^{2}(A, \lambda) x, y\right)\right| \leqslant m /(\operatorname{Re} \lambda)^{2}, \lambda \in \Omega\left(b ; r_{0}\right)$. Отсюда и из равенства $d / d \lambda R(A, \lambda)=R^{2}(A, \lambda), \operatorname{Re} \lambda>0$, нетрудно получить соотношение (см. [5])

$$
(R(A, \lambda) x, y)=-\int_{0}^{\infty}\left(R^{2}(A, s+\lambda) x, y\right) d s .
$$

В силу (7) и (4) имеем

$$
|(R(A, \lambda) x, y)| \leqslant \int_{-\infty}^{\infty}\left\{\int_{0}^{\infty} \frac{d s}{|i t-s-\lambda|^{2}}\right\} d \mu(t), \quad \lambda \in \Omega\left(b ; r_{0}\right) .
$$

При этом $|i t-s-\lambda|^{2} \geqslant s^{2}+|\lambda-i t|^{2}, \operatorname{Re} \lambda>0, s>0$, и тогда

$$
\int_{0}^{\infty} \frac{d s}{|i t-s-\lambda|^{2}} \leqslant \int_{0}^{\infty} \frac{d s}{s^{2}+|i t-\lambda|^{2}}=\frac{\pi}{2|i t-\lambda|},
$$

откуда и следует оценка (6). Лемма доказана.

Далее при использовании теоремы вложения Карлесона [6, гл. 2, п. 3] нам понадобится следующее простое утверждение.

Лемма 2. Пусть $Q(h)$ - произвольный квадрат в плоскости $\mathbb{C}$ со стороной, равной $h$, и $C\left(z_{0} ; \rho\right)$ - окружсность $\left\{z:\left|z-z_{0}\right|=\rho\right\}$. Тогда для длинь $l(\gamma)$ кривой $\gamma=C\left(z_{0} ; \rho\right) \cap Q(h)$ справедлива оченка $l(\gamma) \leqslant 2 \pi h$.

ДокАзАтельство. Достаточно рассмотреть случай $\rho>h$. Пусть центр окружности $z_{0}$ лежит вне $Q(h)$ либо на границе этого квадрата и $z_{1}, z_{2}$ - точки, принадлежащие $\gamma$, такие, что $\gamma$ находится на дуге окружности $z_{1} z_{2}$, и пусть $\alpha \in(0, \pi)$ - угол между отрезками $z_{0} z_{1}$ и $z_{0} z_{2}$. Тогда $\sin (\alpha / 2)=\left|z_{1}-z_{2}\right| /(2 \rho)$, $\left|z_{1}-z_{2}\right| \leqslant \sqrt{2} h$ и, значит,

$$
l(\gamma) \leqslant \alpha \rho \leqslant \pi \rho \sin (\alpha / 2) \leqslant \frac{\pi h}{\sqrt{2}}<2 \pi h .
$$

Пусть $z_{0}$ лежит внутри $Q(h)$ и $Q(h)=\bigcup Q_{j}(h / 2), j=1,2,3,4$. Тогда, не уменьшая общности, можно считать, что $z_{0} \in Q_{1}(h / 2)$ и (в силу того, что $h<\rho$ ) 
$C\left(z_{0} ; \rho\right) \cap Q_{1}(h / 2)=\varnothing$. Используя оценку (8) для $\gamma_{j}=C\left(z_{0} ; \rho\right) \cap Q_{j}(h / 2), j \neq 1$, получаем $l(\gamma) \leqslant l\left(\gamma_{2}\right)+l\left(\gamma_{3}\right)+l\left(\gamma_{4}\right) \leqslant 3 \pi h /(2 \sqrt{2})<2 \pi h$. Лемма доказана.

Теорема. Пусть резольвента оператора $A \in \mathscr{G}$ удовлетворяет условиям (3), (4) и существует оператор $A^{-1} \in E$. Тогда $A^{-1} \in \mathscr{G}$.

ДоказАтельСтво. Согласно замечанию, считаем, что оценка (4) выполняется при $\lambda \in \Omega\left(b ; r_{0}\right)$. Для доказательства теоремы проверим, что резольвента оператора $A^{-1}$ для любых $x \in B, y \in B^{*}$ удовлетворяет условию (5). На основании равенства $(2)$, справедливого при $\operatorname{Re} \lambda>0$, имеем

$\left|\left(R^{2}\left(A^{-1}, \lambda\right) x, y\right)\right| \leqslant|\lambda|^{-2}\|x\|\|y\|_{*}+2|\lambda|^{-3}\left|\left(R\left(A, \lambda^{-1}\right) x, y\right)\right|+|\lambda|^{-4}\left|\left(R^{2}\left(A, \lambda^{-1}\right) x, y\right)\right|$,

и, значит,

$$
\sigma \int_{\sigma-i \infty}^{\sigma+i \infty}\left|\left(R^{2}\left(A^{-1}, \lambda\right) x, y\right)\right||d \lambda| \leqslant \pi\|x\|\|y\|_{*}+\sigma J_{\sigma}, \quad \sigma>0,
$$

где

$$
J_{\sigma}=\int_{\sigma-i \infty}^{\sigma+i \infty}\left\{2|\lambda|^{-3}\left|\left(R\left(A, \lambda^{-1}\right) x, y\right)\right|+|\lambda|^{-4}\left|\left(R^{2}\left(A, \lambda^{-1}\right) x, y\right)\right|\right\}|d \lambda| .
$$

Положим

$$
\Omega_{0}\left(b ; r_{0}\right)=\{\lambda \in \mathbb{C}: \operatorname{Re} \lambda>0\} \backslash \Omega\left(b ; r_{0}\right)=\left\{\lambda \in \mathbb{C}: \operatorname{Re} \lambda>0,|\lambda \pm i b| \leqslant r_{0}\right\}
$$

и сделаем в интеграле $J_{\sigma}$ замену переменной интегрирования $z=1 / \lambda$. Тогда прямая $\operatorname{Re} \lambda=\sigma$ перейдет в окружность $C_{\sigma}=\{z:|z-\varepsilon|=\varepsilon\}, \varepsilon=1 /(2 \sigma)$, а интеграл (с учетом (4), замечания и леммы 1) примет вид

$$
\begin{aligned}
J_{\sigma} & =\int_{C_{\sigma}}\left\{2|z||(R(A, z) x, y)|+|z|^{2}\left|\left(R^{2}(A, z) x, y\right)\right|\right\}|d z| \\
& \leqslant J_{0, \sigma}+\frac{\pi}{2} \int_{-\infty}^{\infty}\left\{\int_{C_{\sigma}}\left\{\frac{2|z|}{|i t-z|}+\frac{|z|^{2}}{|i t-z|^{2}}\right\}|d z|\right\} d \mu(t),
\end{aligned}
$$

где

$$
J_{0, \sigma}=\int_{\gamma_{\sigma}}\left\{2|z||(R(A, z) x, y)|+|z|^{2}\left|\left(R^{2}(A, z) x, y\right)\right|\right\}|d z|, \quad \gamma_{\sigma}=C_{\sigma} \cap \Omega_{0}\left(b ; r_{0}\right) .
$$

При этом справедливо неравенство

$$
\frac{2|z|}{|i t-z|}+\frac{|z|^{2}}{|i t-z|^{2}} \leqslant\left|1+\frac{|z|}{|i t-z|}\right|^{2}=|1+| 1-\frac{i t}{i t-z}||^{2} \leqslant 8+\frac{2 t^{2}}{|i t-z|^{2}}
$$

и оценка интеграла

$$
\int_{C_{\sigma}}\left\{8+\frac{2 t^{2}}{|i t-z|^{2}}\right\}|d z| \leqslant 16 \pi \varepsilon+2 \tau^{2} \varepsilon \int_{0}^{2 \pi} \frac{d \phi}{(\tau-\sin \phi)^{2}+(1+\cos \phi)^{2}}=\frac{10 \pi}{\sigma},
$$

где $\tau=t / \varepsilon=2 \sigma t \in \mathbb{R}$.

Рассмотрим интеграл $J_{0, \sigma}$, когда $\gamma_{\sigma}$ не является пустым множеством, т. е. можно считать, что $\sigma \in\left(0, \sigma_{1}\right)$ для некоторого $\sigma_{1}>0$, зависящего от $b$ и $r_{0}$. При этом $|z| \leqslant b+r_{0}, z \in \gamma_{\sigma}$, и нетрудно заключить, что найдутся такие постоянные $c_{1}>0, c_{2}>0$, для которых при всех $\sigma \in\left(0, \sigma_{1}\right)$ выполняются неравенства

$$
l\left(\gamma_{\sigma}\right) \leqslant c_{1}, \quad \operatorname{Re} z \geqslant c_{2} \sigma \quad \text { для любого } z \in \gamma_{\sigma} .
$$


Так как $A \in \mathscr{G}$, то $\|R(A, z)\| \leqslant M /(\operatorname{Re} z), \operatorname{Re} z>0[1$, гл. $9, \S 1.4]$ и, значит, в силу (10)

$$
\sigma \int_{\gamma_{\sigma}}|z||(R(A, z) x, y)||d z| \leqslant c_{3}, \quad \sigma \in\left(0, \sigma_{1}\right) .
$$

Далее, как отмечалось в начале разд. 2 , при любых $x \in B, y \in B^{*}$ для функции $\left(R^{2}(A, \lambda) x, y\right), \operatorname{Re} \lambda>0$, выполняется оценка (5). Это означает, что для любого $\lambda_{0}>0$ аналитическая при $\operatorname{Re} \lambda>0$ функция $\left(R^{2}\left(A, \lambda+\lambda_{0}\right) x, y\right)$ принадлежит пространству Харди $H^{1}$ в полуплоскости $\operatorname{Re} \lambda>0$. Тогда, полагая $\lambda_{0}=c_{2} \sigma$, где $c_{2}$ - константа из (10), и используя теорему вложения Карлесона, получаем оценку

$$
\sigma \int_{\gamma_{\sigma}}|z|^{2}\left|\left(R^{2}(A, z) x, y\right)\right||d z| \leqslant c_{4} \sigma \int_{\lambda_{0}-i \infty}^{\lambda_{0}+i \infty}\left|\left(R^{2}(A, \lambda) x, y\right)\right||d \lambda| \leqslant c_{5}
$$

с постоянной $c_{5}>0$, не зависящей от $\sigma \in\left(0, \sigma_{1}\right)$. Здесь использовался тот факт, что константа вложения Карлесона $N$, определяемая неравенством

$$
l\left(\gamma_{\sigma} \cap\left\{\operatorname{Re} \lambda \in\left(\lambda_{0}, \lambda_{0}+h\right), \operatorname{Im} \lambda \in(t, t+h)\right\}\right) \leqslant N h, \quad h>0, t \in \mathbb{R},
$$

согласно лемме 2 , допускает оценку $N \leqslant 2 \pi$, не зависящую от $\sigma \in\left(0, \sigma_{1}\right)$.

Таким образом, на основании (9) и проведенных оценок интеграла $J_{\sigma}, \sigma \in$ $\left(0, \sigma_{1}\right)$, заключаем, что для $\left(R^{2}\left(A^{-1}, \lambda\right) x, y\right)$ при любых $x \in B, y \in B^{*}$ выполняется условие (5), и, значит, $A^{-1} \in \mathscr{G}$. Теорема доказана.

СледСтвиЕ 1. Пусть для резолъвенты оператора $A \in \mathscr{G}$ выполняется оцен$\kappa a$

$$
\|R(A, \lambda)\| \leqslant c \sum_{k=1}^{n} \frac{1}{\left|i a_{k}-\lambda\right|}, \quad \operatorname{Re} \lambda>0,
$$

с некоторой постоянной $c>0$ и действительными числами $a_{k}, k=1, \ldots, n$. Тогда если существует оператор $A^{-1} \in E$, mо $A^{-1} \in \mathscr{G}$.

Утверждение следствия вытекает из теоремы, где условие (4) выполняется при $\operatorname{Re} \lambda>0$ с мерой

$$
\mu=c^{2} n\|x\|\|y\|_{*} \sum_{k=1}^{n} \delta_{a_{k}},
$$

сосредоточенной в точках $t=a_{k}$.

СледСТВиЕ 2. Пусть $B=H$ - сепарабельное гильбертово пространство и резольвента оператора $A \in \mathscr{G}(H)$ удовлетворяет условию (4). Тогда если ker $A=\{0\}$, mo $A^{-1} \in \mathscr{G}(H)$.

В случае гильбертова пространства из условий $A \in \mathscr{G}(H)$, $\operatorname{ker} A=\{0\}$ следует, что $A^{-1} \in E(H)$ [7]. Таким образом, утверждение $A^{-1} \in \mathscr{G}(H)$ вытекает из теоремы с учетом того, что для оператора $A \in \mathscr{G}(H)$ условие (3) выполняется автоматически.

Условию (4) удовлетворяет произвольный оператор $A \in E(B)$ с оценками резольвенты

$$
\|R(A, \lambda)\| \leqslant \frac{c}{|\lambda|}, \quad \operatorname{Re} \lambda>0,|\lambda| \in\left(0, r_{2}\right) \cup\left(r_{1}, \infty\right), r_{1} \geqslant r_{2}>0 .
$$


В частности, если $A$ является ограниченным и ограниченно обратимым в $B$ оператором, то для резольвенты $R(A, \lambda)$ справедливо условие (11) для (например) $r_{2}=\left(2\left\|A^{-1}\right\|\right)^{-1}, r_{1}=2\|A\|$, причем при $|\lambda| \leqslant\left(2\left\|A^{-1}\right\|\right)^{-1}$ резольвента $R(A, \lambda)$ является ограниченной оператор-функцией.

Отметим, что оценка (11) при $\operatorname{Re} \lambda>0,|\lambda| \in\left(r_{1}, \infty\right)$ эквивалентна тому, что $A \in \mathscr{H}_{0}$, а с другой стороны, если $A^{-1} \in E$, то оценка (11) при $\operatorname{Re} \lambda>0$, $|\lambda| \in\left(0, r_{2}\right)$ эквивалентна условию $A^{-1} \in \mathscr{H}_{0}$ (см. [1, гл. $\left.\left.9, \S 1.7\right]\right)$. Таким образом, на основании следствия 2 получаем такое утверждение:

СлеДСТВИЕ 3. Пусть $B=H$ - сепарабельное гильбертово пространство и $A \in \mathscr{H}_{0}, A^{-1} \in \mathscr{H}_{0}$. Тогда справедливо утверждение

$$
A \in \mathscr{G} \Longleftrightarrow A^{-1} \in \mathscr{G} .
$$

$B$ частности, если $A$ является ограниченным и ограниченно обратимым $в$ $H$ оператором, то из включения $A \in \mathscr{G}$ вытекает включение $A^{-1} \in \mathscr{G} u$ наоборот.

Отметим, что, в отличие от случая $A \in \mathscr{H}(\theta)$, из выполнения условий $A \in$ $\mathscr{G} \cap \mathscr{H}_{0}, A^{-1} \in \mathscr{G}$ в общем случае не вытекает включение $A^{-1} \in \mathscr{H}_{0}$. Действительно, в пространстве $H=L_{2}[0,1]$ оператор интегрирования $(J f)(s)=$ - $\int_{0}^{s} f(\tau) d \tau$ принадлежит $\mathscr{G} \cap \mathscr{H}_{0}$, в то же время обратный оператор $J^{-1} \in \mathscr{G}$, $\left(J^{-1} f\right)(s)=-f^{\prime}(s)$, с областью определения $\mathscr{D}\left(J^{-1}\right)=\left\{f \in W_{2}^{1}[0,1]: f(0)=0\right\}$ порождает в $L_{2}[0,1]$ полугруппу сдвигов $\left(e^{t J^{-1}} f\right)(s)=f(s-t), s \in(t, 1)$, $t \in(0,1),\left(e^{t J^{-1}} f\right)(s)=0, s \in(0,1) \cap(0, t)$, которая не является даже дифференцируемой (при $t \in(0,1))$ полугруппой операторов.

3. В заключение отметим, что рассмотрение задачи, которой посвящена эта работа, обусловлено как внутренней логикой развития теории полугрупп операторов (функциональное исчисление для генераторов полугрупп операторов, cм., например, $[2$, гл. 8, §2], а также [8-10]), так и естественными приложениями, связанными с вопросами разрешимости задачи Коши для абстрактных дифференциальных уравнений первого порядка, не разрешенных относительно производной (см. [11] и имеющуюся там библиографию). Так, например, если $A \in \mathscr{G}(B), A^{-1} \in \mathscr{G}(B)$, то можно показать, что и оператор $L=\alpha A+A^{-1}$ с $D(L)=D(\alpha A) \cap D\left(A^{-1}\right)$ при любом $\alpha \geqslant 0$ принадлежит $\mathscr{G}(B)$, причем (см. $[12, \S 2]) e^{t L}=e^{t \alpha A} e^{t A^{-1}}=e^{t A^{-1}} e^{t \alpha A}, t \geqslant 0$. Отсюда нетрудно заключить (см. [1, гл. 9]), что задача Коши

$$
\frac{d A u(t)}{d t}=\left(\alpha A^{2}+I\right) u(t), \quad t \geqslant 0, u(0)=u_{0} \in B,
$$

для любого $u_{0} \in D\left(A^{2}\right)$ имеет единственное ограниченное при $t \geqslant 0$ решение $u(t)=e^{t L} u_{0}$. Если $\alpha=0$, то последнее утверждение справедливо при любом $u_{0} \in D(A)$.

Автор выражает благодарность Т. Я. Азизову, привлекшему его внимание к рассмотренной в этой работе тематике.

\section{ЛИТЕРАТУРА}

1. Kaто T. Теория возмущений линейных операторов. Мир, М., 1972.

2. Данфорд Н., Швари, Дж. Т. Линейные операторы. Общая теория. ИЛ., М., 1962. 
3. de Laubenfels R. Inverses of generators. Proc. Amer. Math. Soc., 104, No. 2, 443-448 (1988).

4. Горбачук М. Л., Мацищин И. Т. Поведение на бесконечности решений дифференциального уравнения первого порядка параболического типа в банаховом пространстве. ДАН СССР, 312, №3, 521-524 (1990).

5. Гомилко A. M. Об условиях на производящий оператор равномерно ограниченной $C_{0}$-полугруппы операторов. Функц. анализ и его прил., 33, вып. 4, 66-69 (1999).

6. Гарнетm Дж. Ограниченные аналитические функции. Мир, М., 1984.

7. Goldstein J. A. Semigroups of linear operators and applications. Oxford Univ. Press, New York, 1985.

8. de Laubenfels $R$. Powers of generators of holomorphic semigroups. Proc. Amer. Math. Soc., 99, No. 1, 105-108 (1987).

9. Горбачук В. И., Князюк A. В. Граничные значения решений дифференциальнооператорных уравнений. УМН, 44, вып. 3, 55-91 (1989).

10. Миротин A. P. О $\mathscr{T}$-исчислении генераторов $C_{0}$-полугрупп. Сиб. матем. ж., 39 , No. 3, 571-582 (1998).

11. Свиридюк Г. А. К общей теории полугрупп операторов. УМН, 49, вып. 4, 47-79 (1994).

12. Васильев B. В., Крейн С. Г., Пискарев С. И. Полугруппы операторов, косинус оператор-функции и линейные дифференциальные уравнения. Итоги науки и техники. Математический анализ. Т. 28, ВИНИТИ, М., 1990, с. 87-202.

Институт гидромеханики НАН Украины, Киев email: gomilko@ukrpost.net
Поступило в редакцию 11 августа 2003 г. 